\title{
Regulation and evolution of flavonoid biosynthesis pathway in polyploid plants
}

\author{
Strygina K.V. ${ }^{1,2 *}$, Khlestkina E.K. ${ }^{1,2}$ \\ ${ }^{1}$ N.I. Vavilov All-Russian Institute of Plant Genetic Resources (VIR), St. Petersburg, Russia \\ ${ }^{2}$ Institute of Cytology and Genetics, SB RAS, Novosibirsk, Russia \\ *e-mail:k.strygina@vir.nw.ru
}

Higher plants, including cereals, synthesize secondary metabolites flavonoids. The regulation of the expression of flavonoid biosynthesis genes is under control of genetic and epigenetic mechanisms. Genetic regulation occurs with the MBW complex, which is formed due to the action of the Myb, bHLH and WD40 transcription factors (TF), while DNA methylation is important for the binding of TF with cis-regulatory genes regions. The aim of this work was the characterisation of $M y b, b H L H$ and WD40 gene copies in the Triticeae tribe, on the one hand, and the studying of the methylation patterns of promoters of flavonoid biosynthesis genes in wheat genome, on the other hand. In this work, we identified and characterized in the genomes of the Triticeae tribe $b H L H$ gene copies in 2 and 4 groups of chromosomes, $M y b$ gene copies in 4 and 7 groups of chromosomes and WD40 gene copies in 6 chromosomes. A study of the structure organisation and transcriptional activity revealed the full range of regulatory MBW genes controlling the synthesis of anthocyanins in the pericarp and aleurone layer of wheat and barley. We demonstrated that bHLH-coding gene $H v M y c 2$ is the main regulator of the appearance of blue colour in barley grain. The bHLH-coding candidate gene TaMyc-B1 determining the colour of the wheat coleoptile was also detected. In addition, analysis of methylation patterns of promoters of flavonoid biosynthesis genes in wheat showed that the methylation does not make a significant contribution in the specific expression pattern of the studied genes. In general, the results of the comparison of the flavonoid biosynthesis genes copies demonstrate that the maintenance of their functional state is the cause of their tissue-specific activity in members of the Triticeae tribe.

Acknowledgements: This study was partially supported by RFBR (No. 18-416-543007). 